\title{
Polyubiquitin Is Required for Growth, Development and Pathogenicity in the Rice Blast Fungus Magnaporthe oryzae
}

\author{
Yeonyee $\mathrm{Oh}^{1}$, William L. Franck ${ }^{1}$, Sang-Oh Han $^{2}$, Angela Shows' ${ }^{1}$, Emine Gokce ${ }^{3}$, David C. Muddiman ${ }^{3}$, \\ Ralph A. Dean ${ }^{1 *}$
}

1 Center for Integrated Fungal Research, Department of Plant Pathology, North Carolina State University, Raleigh, North Carolina, United States of America, 2 Department of Medicine, Duke University, Durham, North Carolina, United States of America, 3 W. M. Keck FT-ICR Mass Spectrometry Laboratory, Department of Chemistry, North Carolina State University, Raleigh, North Carolina, United States of America

\begin{abstract}
Protein ubiquitination, which is highly selective, regulates many important biological processes including cellular differentiation and pathogenesis in eukaryotic cells. Here, we integrated pharmacological, molecular and proteomic approaches to explore the role of ubiquitination in Magnaporthe oryzae, the leading fungal disease of rice world-wide. Inhibition of ubiquitin-mediated proteolysis using the $26 \mathrm{~S}$ proteasome inhibitor, Bortezomib, significantly attenuated conidia germination, appressorium formation and pathogenicity in M. oryzae. Gene expression analysis revealed that many genes associated with protein ubiquitination were developmentally regulated during conidia germination. Only a few, including a polyubiquitin encoding gene, MGG_01282, were more abundantly expressed during appressorium formation and under nitrogen starvation. Targeted gene deletion of MGG_01282, in addition to a significant reduction in protein ubiquitination as determined by immuno blot assays, resulted in pleiotropic effects on $M$. oryzae including reduced growth and sporulation, abnormal conidia morphology, reduced germination and appressorium formation, and the inability to cause disease. Mutants were also defective in sexual development and were female sterile. Using mass spectrometry, we identified 63 candidate polyubiquitinated proteins under nitrogen starvation, which included overrepresentation of proteins involved in translation, transport and protein modification. Our study suggests that ubiquitination of target proteins plays an important role in nutrient assimilation, development and pathogenicity of M. oryzae.
\end{abstract}

Citation: Oh Y, Franck WL, Han S-O, Shows A, Gokce E, et al. (2012) Polyubiquitin Is Required for Growth, Development and Pathogenicity in the Rice Blast Fungus Magnaporthe oryzae. PLoS ONE 7(8): e42868. doi:10.1371/journal.pone.0042868

Editor: Kirsten Nielsen, University of Minnesota, United States of America

Received May 18, 2012; Accepted July 12, 2012; Published August 10, 2012

Copyright: $\subset 2012$ Oh et al. This is an open-access article distributed under the terms of the Creative Commons Attribution License, which permits unrestricted use, distribution, and reproduction in any medium, provided the original author and source are credited.

Funding: This work was supported through National Science Foundation Award \#0918611 and \#0605017. The funder had no role in study design, data collection and analysis, decision to publish, or preparation of the manuscript.

Competing Interests: The authors have declared that no competing interests exist.

*E-mail: radean2@ncsu.edu

\section{Introduction}

Ubiquitin mediated protein degradation is a highly conserved process and plays important roles in a variety of cellular processes, including transcriptional regulation, signal transduction, cell cycling, cellular differentiation and pathogenesis [1]. Ubiquitin, a highly conserved 76-amino acid protein, is activated by the ubiquitin activating enzyme E1 using ATP, which is then transferred to a ubiquitin conjugating enzyme E2. The E2 enzyme and the protein substrate bind specifically to a particular ubiquitinprotein ligase E3 resulting in the carboxy-terminal glycine of ubiquitin becoming covalently attached to a lysine residue of the protein substrate through an iso-peptide bond. The specificity of targeted proteins is largely controlled by E3 ligases. Successive conjugation of ubiquitin generates a polyubiquitin chain that is recognized by regulatory particles in the proteasome for degradation or as a trigger for various signaling pathways [1].

Ubiquitin is one of the most abundant cellular proteins, representing $1-5 \%$ of total cellular protein $[2,3]$. Ubiquitin levels are maintained by recycling of ubiquitin from ubiquitin substrate conjugates by deubiquitinating enzymes (DUBs) and de novo ubiquitin synthesis through transcriptional regulation. In yeast, ubiquitin is produced by cleavage from precursor proteins, where ubiquitin is fused to unrelated peptide sequences (UBI1, UBI2 and $U B I 3$ ) or to itself (UBI4) [4,5]. UBI4 encodes a polyubiquitin protein that contains five consecutive ubiquitin repeats and is highly induced under stress conditions [4,5]. Ubiquitination appears to play an important role in host-pathogen interactions. In a number of plant pathogenic fungi, polyubiquitin transcript levels significantly increased during in planta colonization or under environmental stress [6-8]. In the human pathogen Candida albicans, inactivation of polyubiquitin gene UBI4 affected fungal growth, stress resistance and virulence [9]. However, direct evidence for the role of ubiquitination in plant pathogenic fungi is lacking.

Rice blast is the most important disease of rice worldwide, and is caused by the filamentous ascomycete fungus, Magnaporthe oryzae [10]. Common to many other phytopathogenic fungi, M. oryzae elaborates a specialized infection cell, the appressorium to infect its host [11]. Perception of environmental cues [12], starvation responses [13], cell signaling pathways [12,14,15], turgor pressure generation [16], recycling cellular contents (autophagy) [17] and 
A
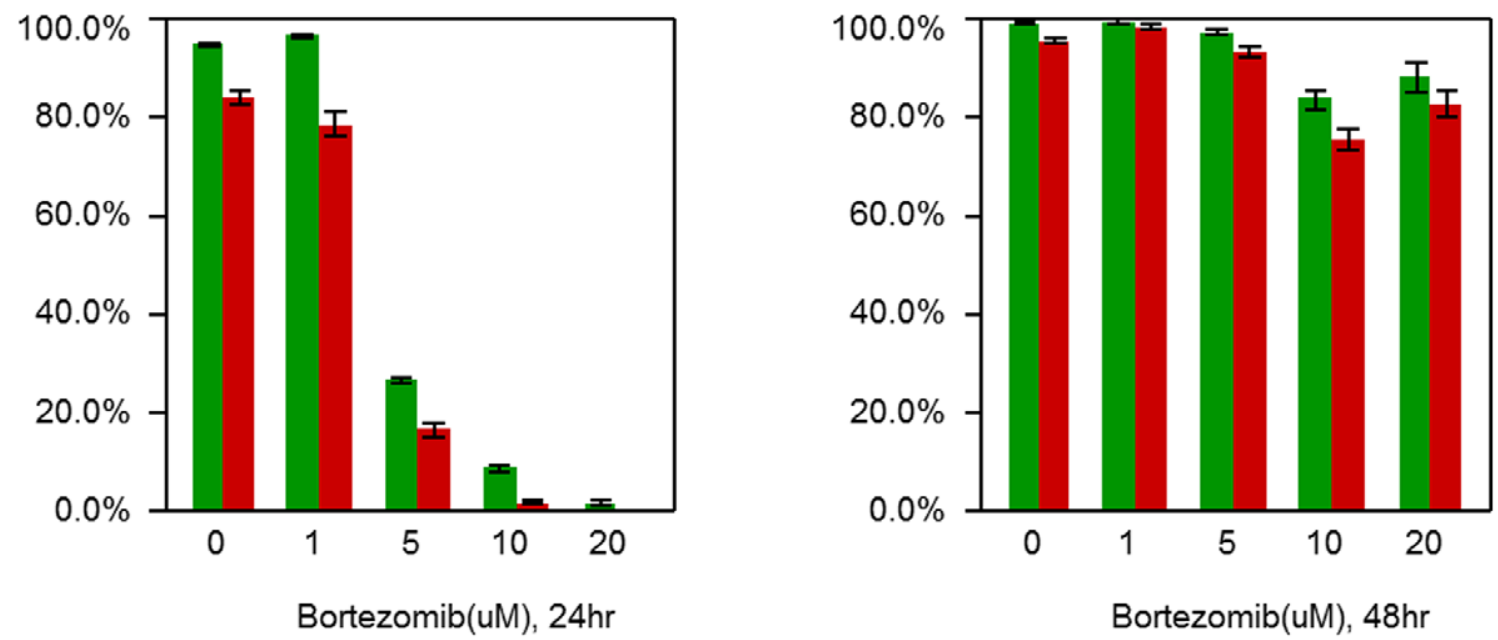

B

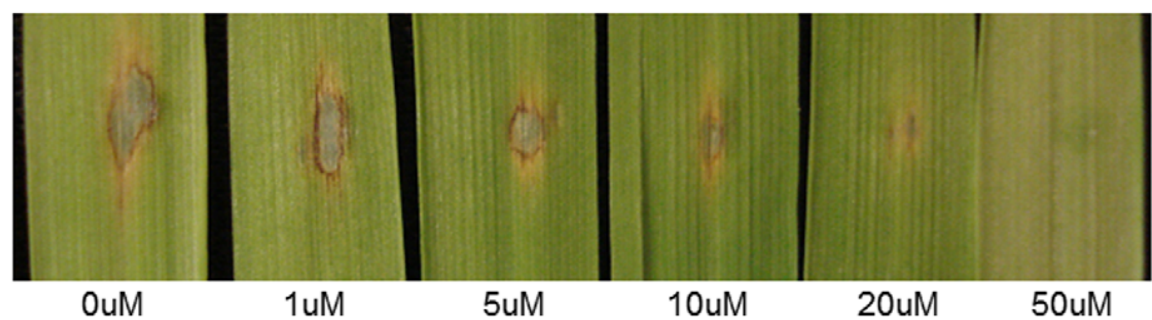

Figure 1. Bortezomib blocks conidia germination, appressorium formation and pathogenicity. A. Conidia germination and appressorium formation (green and red bar respectively) were measured after 24 and $48 \mathrm{hr}$ incubation on a hydrophobic surface. B. Pathogenicity assays were performed on 6 day old barley leaves. Disease progress was assessed after 5 day incubation.

doi:10.1371/journal.pone.0042868.g001

cell cycle checkpoints [18] are known to orchestrate the development of this specialized cell. Through global gene expression and functional analyses, we previously identified a connection between protein turnover and the infection process. Genes including SPM1, a vacuolar protease and MGD1, a putative $\mathrm{NAD}(+)$ dependent glutamate dehydrogenase (required for recycling carbon and nitrogen from amino acids back into metabolism) were up regulated during appressorium formation. Deletion of either gene resulted in defective appressoria and greatly reduced ability to cause disease [19]. We also observed the transcripts of polyubiquitin gene (MGG_01282) to be significantly enriched during appressorium development as well as under nitrogen starvation conditions [13,19]. In this study, we conducted a comprehensive investigation of the role of protein ubiquitination in $M$. oryzae using pharmacological, molecular and proteomic approaches. Our findings suggest that ubiquitin-mediated proteolysis, which is known to be highly selective, plays a key role in nutrient assimilation, fungal development and pathogenicity of M. oryzae.

\section{Materials and Methods}

Effect of a Proteasome Inhibitor, Bortezomib, on M. oryzae

Wild type $M$. oryzae strain 70-15 was used for all experiments unless otherwise indicated. Conidia were collected from solidified V8 medium after 8 days in water and adjusted to $5 \times 10^{4}$ conidia/ $\mathrm{ml}$. Bortezomib (LC laboratories) stock was prepared in DMSO
( $4 \mathrm{ug} / \mathrm{ul}$ ) and added to the conidial suspension to a bring the final concentration up to $50 \mathrm{uM}$. $50 \mathrm{ul}$ of conidial suspension was placed on the appressorium inducing hydrophobic surface of Gelbond film (Lonza). After 24 and $48 \mathrm{hr}$ incubation, conidia germination and appressorium formation were assessed. For infection assays, $10 \mathrm{ul}$ of conidial suspension in Bortezomib was spotted onto detached 6 day old barley (ROBUST) leaves, placed in a humid plastic container and disease progress monitored for 5 days. Experiments were repeated three times with 3 replicates.

Prediction of Ubiquitin Associated Proteins in M. oryzae and their Transcription Analysis

Ubiquitin associated (UA) InterPro domains were selected based on other studies [20]. M. oryzae proteins with UA Interpro domains were extracted from European Bioinformatics Institute (EBI) Interpro databases (http://www.ebi.ac.uk/interpro/) using the Biomart filtering tool and were categorized according to function in the ubiquitination pathway. Gene expression for UA proteins during fungal development and nitrogen starvation were extracted from the previously reported genome-wide $M$. oryzae microarray data (NCBI GenBank: GSE1945, GSE10173 and GSE 2716).

\section{Targeted Gene Replacement and Complementation of MGG_01282}

Gene replacement cassettes were constructed using adaptamer mediated PCR as previously described [21]. Briefly about $1 \mathrm{~kb}$ of upstream and downstream sequence of MGG_01282 gene was 
Table 1. Ubiquitin pathway associated proteins in M. oryzae.

\begin{tabular}{|c|c|c|c|c|}
\hline \multirow{2}{*}{$\begin{array}{l}\text { Class } \\
\text { UA }\end{array}$} & & \multicolumn{2}{|c|}{ InterPro domain } & \multirow{2}{*}{$\begin{array}{l}\text { \# of proteins } \\
12\end{array}$} \\
\hline & & IPR000626 & Ubiquitin & \\
\hline & & IPR004854 & Ubiquitin fusion degradation protein UFD1 & 2 \\
\hline & & IPR001012 & UBX & 6 \\
\hline & & IPR003892 & Ubiquitin system component Cue & 6 \\
\hline & & IPR000449 & Ubiquitin-associated/translation elongation factor EF1B, N-terminal & 7 \\
\hline & & IPR003903 & Ubiquitin interacting motif & 13 \\
\hline & UA total & & & 44 \\
\hline \multirow[t]{4}{*}{ E1 } & & IPR000011 & Ubiquitin-activating enzyme, E1-like & 2 \\
\hline & & IPR000127 & Ubiquitin-activating enzyme repeat & 3 \\
\hline & & IPR000594 & UBA/THIF-type NAD/FAD binding fold & 8 \\
\hline & E1 total & & & 8 \\
\hline \multirow[t]{2}{*}{ E2 } & & IPR000608 & Ubiquitin-conjugating enzyme, E2 & 21 \\
\hline & E2 total & & & 21 \\
\hline \multirow[t]{8}{*}{ E3 } & & IPR001232 & SKP1 component & 1 \\
\hline & & IPR003126 & Zinc finger, $\mathrm{N}$-recognin & 2 \\
\hline & & IPR003613 & U box domain & 3 \\
\hline & & IPR001373 & Cullin, N-terminal & 4 \\
\hline & & IPR000569 & HECT & 6 \\
\hline & & IPR001810 & F-box domain, cyclin-like & 26 \\
\hline & & IPR001841 & Zinc finger, RING-type & 53 \\
\hline & E3 total & & & 94 \\
\hline \multirow[t]{4}{*}{ DUB } & & IPR001607 & Zinc finger, UBP-type & 3 \\
\hline & & IPR001578 & Peptidase $\mathrm{C} 12$, ubiquitin carboxyl-terminal hydrolase 1 & 4 \\
\hline & & IPR001394 & Peptidase C19, ubiquitin carboxyl-terminal hydrolase 2 & 15 \\
\hline & DUB total & & & 20 \\
\hline Total & & & & 183 \\
\hline
\end{tabular}

amplified with primers that contained adaptamer sequences (see Table S1). A $1.5 \mathrm{~kb}$ fragment containing the hygromycin B phosphotransferase gene (HPH) driven by the trpC promoter from Aspergillus nidulans was amplified from plasmid PCB1003 using the adaptamer sequence attached to the forward HPHF and reverse HPHR primer set. Using nested primers (see Table S1) from inside of the $5^{\prime}$ upstream fragment and from inside of the $3^{\prime}$ end of the downstream fragment of the target gene, the individual fragments and hygromycin resistance gene fragment were combined and amplified together to construct a hygromycin cassette for gene replacement approximately $\sim 3.1 \mathrm{~kb}$ in length. The hygromycin cassette was transformed into 70-15 protoplasts as previously described [22]. Gene replacement mutants were identified by PCR screening and further confirmed by Southern blot analysis using ECL system of oligolabeling and detection (Amersham Co.) (Figure S1). For complementation, a $2.6 \mathrm{~kb}$ DNA fragment corresponding to the MGG_01282 gene and its promoter region was PCR amplified (see Table S1) from 70-15 wild type genomic DNA, and using gateway cloning system was cloned into a modified pDONR221 plasmid in which the Bialophos resistance gene had been inserted. The complementation construct was introduced into mutant protoplasts using standard protocols and transformants were screened on Bialophos at $200 \mu \mathrm{g} / \mathrm{ml}$. The complemented strains were identified by PCR amplification of the insert and further confirmed by the recovery of wild type phenotypes.

\section{Mutant Phenotype Assays}

A series of phenotype analyses were conducted on several knockout mutants $(\geq 3)$ and ectopic $(\geq 2)$ transformants. Germination and appressorium assays were conducted using conidia collected from 8 day old V8 agar plates and adjusted to $5 \times 10^{4}$ conidia $/ \mathrm{ml}$. Conidia suspension was spotted on the hydrophobic and hydrophilic surface of GelBond film and rate of germination and appressorium formation was measured after $24 \mathrm{hr}$ incubation at $25^{\circ} \mathrm{C}$ in the dark. To test for pathogenicity, barley and rice seedlings were spray inoculated with $M$. oryzae conidia suspension $\left(5 \times 10^{4}\right.$ conidia/ml, Tween $\left.200.025 \%\right)$ and incubated in dark humid conditions at $25^{\circ} \mathrm{C}$. The number and size of lesions were evaluated 5 days post-inoculation. Lesions on three leaves were counted for each strain, and this experiment was repeated three times. Wound assays were performed by a pinprick with a sterile needle on detached barley leaves. 5 millimeter square agar blocks from 8 day old culture on $\mathrm{V} 8$ of each strain were directly placed onto the wound site, and incubated in humid chambers as described above. Measurements were taken from nine wound sites (three per leaf) per strain, and this experiment was repeated twice. Disease progress and symptom development of MGG_01282 mutant was compared to wild type, ectopic, complemented strain or control treatment. Growth rate assays were conducted by placing $10 \mathrm{ul}$ conidia suspension $\left(5 \times 10^{4} \mathrm{CO}^{-}\right.$ nidia/ml) on agar plates with complete media, minimal media and minimal media without nitrogen source. Colony morphology and 

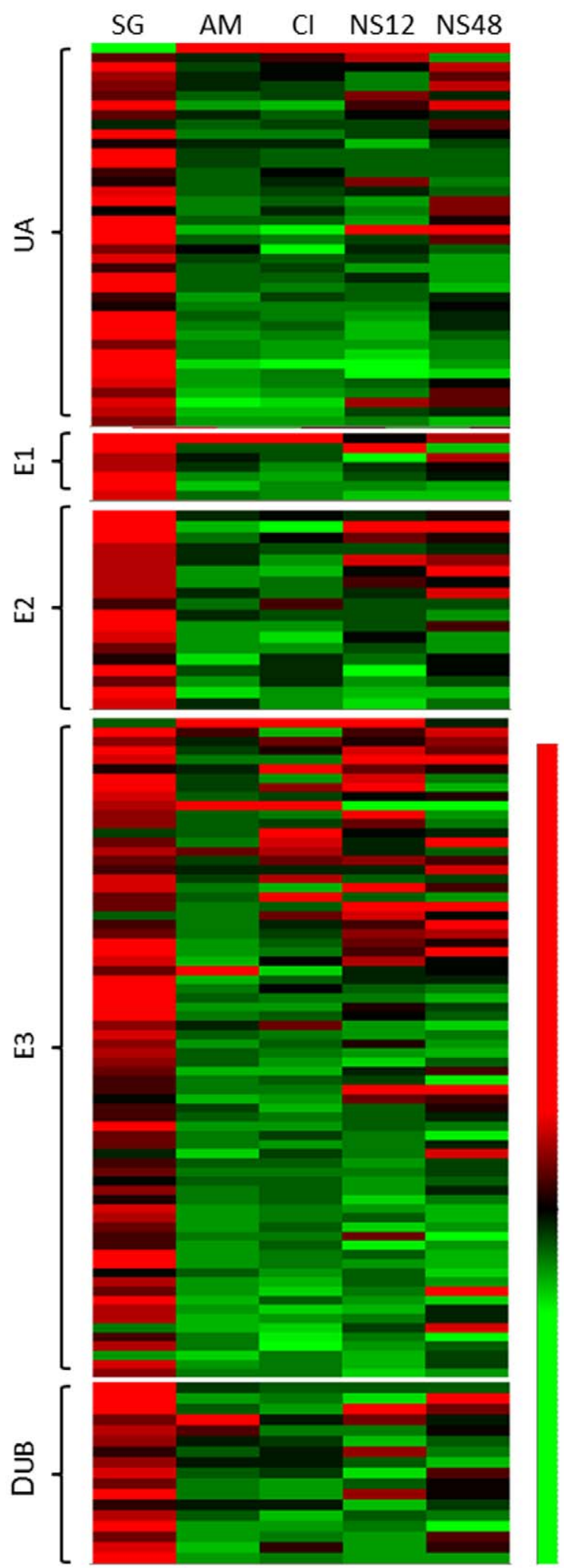

16.2

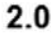

0.5

Figure 2. Ubiquitin associated genes are differentially expressed during conidia germination, appressorium formation and under nitrogen starvation. Each column represents hierarchical clustering profile for spore germination (SG), appressorium maturation (AM), CAMP induced appressoria formation $(\mathrm{Cl})$ and nitrogen starvation for 12 (N12) and 48 (NS48) hr. Genes of same expected function were grouped together as ubiquitin associated (UA), ubiquitin-activating enzymes (E1), ubiquitin-conjugating enzymes (E2), ubiquitin protein ligases (E3) and de-ubiquitinating enzymes (DUB).

doi:10.1371/journal.pone.0042868.g002

diameters were recorded periodically for 15 days. The total number of conidia on minimal media plates was counted after 15 days incubation. Conidia size and morphology were also assessed using a minimum of 100 conidia per replicate. All experiments were conducted in triplicate and performed at least 3 times.

\section{Sexual Reproduction}

M. oryzae 70-15 strains were crossed with $M$. oryzae strain 40915-8, a weeping lovegrass (Eragrostis curvula) pathogen [23] on oatmeal media. Plates were incubated at $25^{\circ} \mathrm{C}$ for 7 days and then incubated at $20^{\circ} \mathrm{C}$ for 21 days under constant light. Plates were examined for fruiting body formation. Perithecia were excised and crushed to identify ascospores under a brightfield microscope.

\section{Western Blotting}

Mycelia samples from wild type, mgg_01282 mutant and ectopic strains were collected from 3 day old liquid minimal media cultures, filtered through miracloth and washed with water. Excess liquid was squeezed out and $500 \mathrm{mg}$ of semi-dried mycelia was ground using liquid nitrogen and resuspended with $2 \mathrm{ml}$ lysis buffer containing $50 \mathrm{mM}$ HEPES ( $\mathrm{pH} 7.5$ ), 0.5\% Nonidet P-40, $250 \mathrm{mM} \mathrm{NaCl}, 10 \%$ (v/v) glycerol, 2 mM EDTA (pH 8.0), and a complete protease inhibitor cocktail (Roche). Protein quantification of each soluble lysate was performed by Bradford assay. $85 \mathrm{ug}$ of each protein sample was separated on a $4-20 \%$ gradient gel (Invitrogen) and transferred to a nitrocellulose membrane. Blots were blocked in 5\% bovine serum albumin (BSA) and antibody incubations were carried out in 5\% skim milk followed by washes. Signals were detected by SuperSignal West Pico Chemiluminescent Substrate (Pierce), following the manufacturer's instructions. The following antibodies were used. P4D1 (1:1000, Cell Signaling Technology), K63 (1:1000, Cell Signaling Technology) and Antimouse IgG (1:3000, Cell Signaling Technology).

\section{Ubiquitinated Protein Profiling by NanoLC-MS/MS}

Wild type $M$. oryzae 70-15 was incubated in liquid complete media for 3 days. Mycelia were collected, washed thoroughly with distilled water and then inoculated into liquid minimal media without nitrogen source. After $12 \mathrm{hr}$ incubation, 3 replicates were pooled, mycelia tissues were collected and proteins extracted. The putative polyubiquitinated protein sample was enriched using Agarose-Tube2 (LIfeSensors) following manufacturer's protocol. To prepare the sample for NanoLC-MS/MS, dithiothreitol (Biorad, Hercules) was added to the protein sample to a final of $5 \mathrm{mM}$ and was incubated for $30 \mathrm{~min}$ at $56^{\circ} \mathrm{C}$ to reduce the protein disulfide bonds. The samples were then mixed with $200 \mu \mathrm{l}$ of $8 \mathrm{M}$ Urea (Sigma Aldrich), loaded onto Vivacon $500 \mu \mathrm{L}$ ultrafiltration spin columns with $30 \mathrm{kDa}$ MW cutoff (Sartorius Stedim Biotech) and centrifuged at $14,000 \times \mathrm{g}$ for $15 \mathrm{~min}$. The columns were washed one more time before Iodoacetamide (Sigma Aldrich) was added to a final of $20 \mathrm{mM}$. The samples were incubated for $30 \mathrm{~min}$ in the dark at room temperature for alkylation of the free thiols. The filter units were then centrifuged at $14,000 \times \mathrm{g}$ for $10 \mathrm{~min}$. Three $100 \mu \mathrm{l} 8 \mathrm{M}$ Urea washes and $30.05 \mathrm{M}$ ammonium bicarbonate (Sigma Aldrich) washes with 15 min subsequent centrifugations were performed. The collection vials were changed and $0.4 \mu \mathrm{g} / \mu \mathrm{l}$ trypsin (Sigma Aldrich) was added at a 1:100 enzyme: protein ratio. Digestion was performed over night at 
A
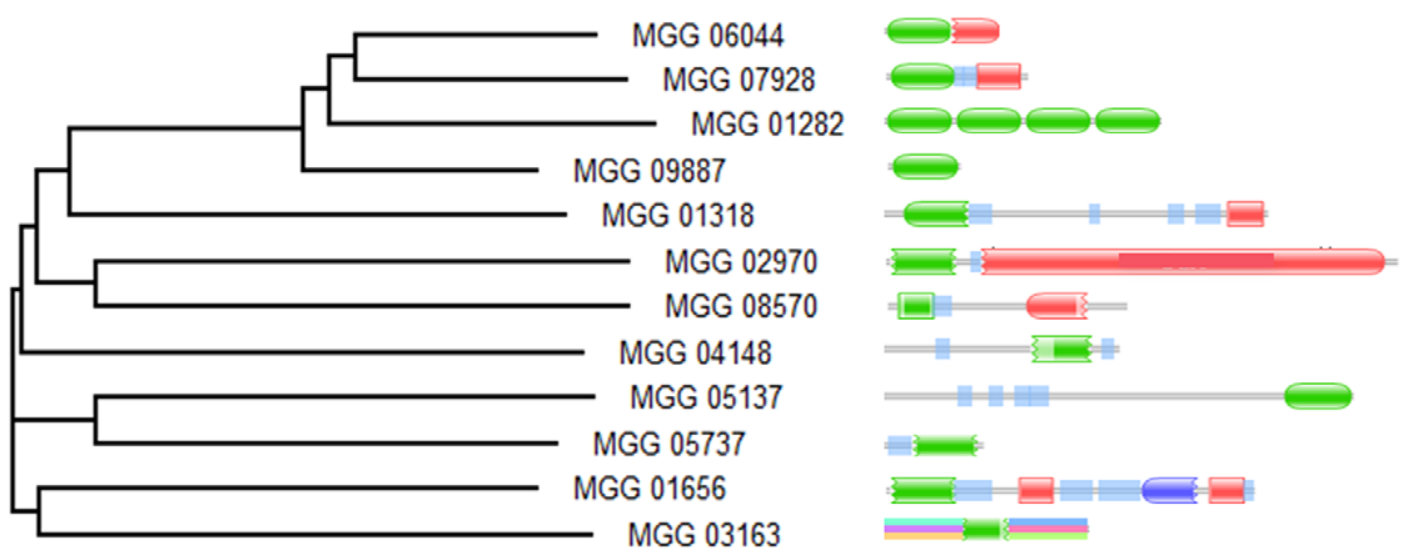

B

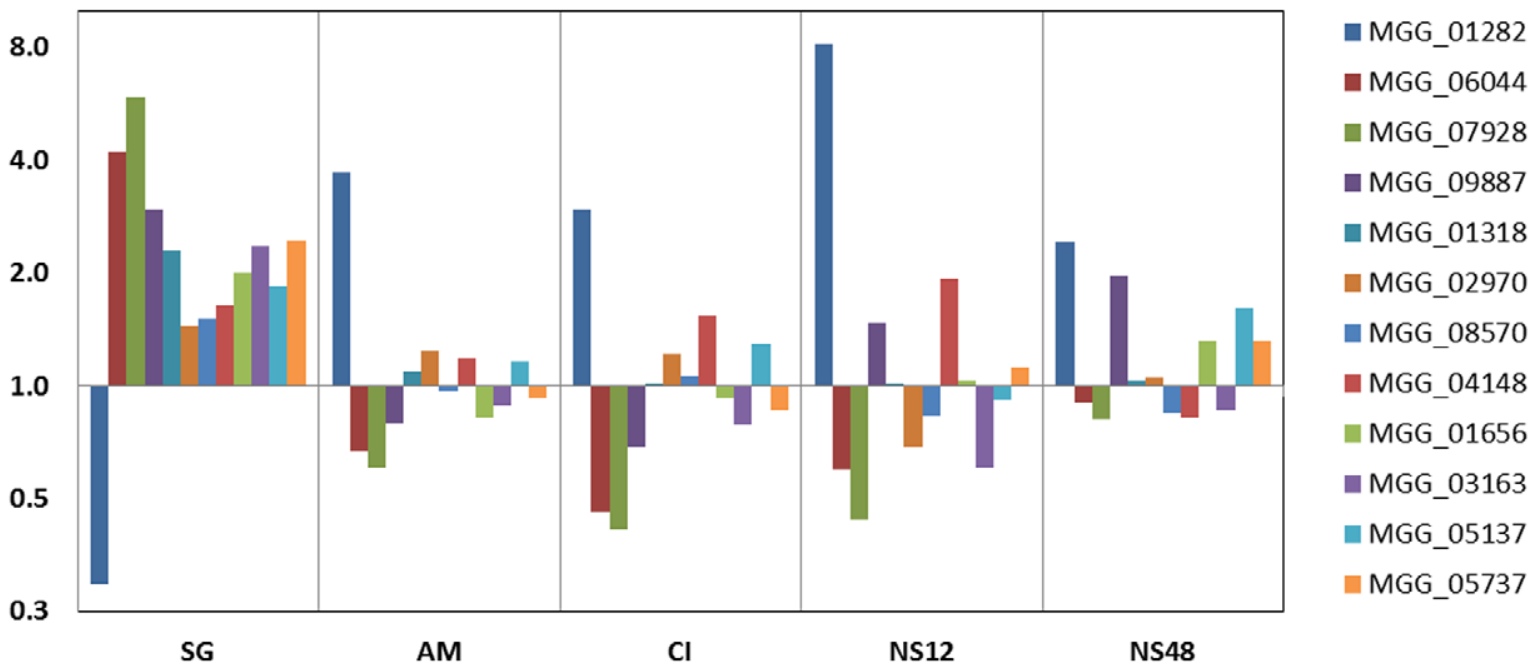

Figure 3. Clustering and gene expression of 12 ubiquitin related proteins in $M$. oryzae. A. Proteins were grouped using ClustalW and functional domains indicated. Green oval structure indicates a ubiquitin moiety and others in green indicate partial or ubiquitin like domains. B. Gene expression during spore germination (SG), appressorium maturation (AM), CAMP induced appressoria formation (CI) and nitrogen starvation for 12 (NS12) and 48 (NS48) hr.

doi:10.1371/journal.pone.0042868.g003

$37^{\circ} \mathrm{C}$ and peptides eluted by adding $40 \mu \mathrm{l}$ ammonium bicarbonate and centrifuging the filter units at $14,000 \times \mathrm{g}$ for $10 \mathrm{~min}$. Each sample was injected 3 times to a LTQ FT Ultra Hybrid Mass Spectrometer and the data analyzed as previously described [24].

\section{Results}

Inhibition of Proteasome Mediated Protein Degradation Blocks Conidial Germination, Appressorium Induction and Pathogenicity in $\mathrm{M}$. oryzae

To investigate the role of ubiquitin mediated proteasomal protein degradation during the infection-related fungal development, we treated conidia with the proteasome inhibitor, Bortezomib. On an appressorium inductive hydrophobic surface, both conidial germination and appressorium formation were significantly delayed in a dose-dependent manner with $20 \mathrm{uM}$ Bortezomib being completely inhibitory after $24 \mathrm{hr}$ incubation. However, at $48 \mathrm{hr}$, most conidia germinated and successfully formed melanized appressoria (Figure 1A, 1B). Bortezomib also inhibited pathogenicity. Compared to typical spreading necrotic lesions on control infected barley leaves, the addition of $1 \mathrm{uM}$ Bortezomib resulted in smaller lesions. Symptom development was completely blocked when the conidia solution contained $50 \mathrm{uM}$ Bortezomib (Figure 1C). $50 \mathrm{uM}$ Bortezomib solution had no observable effect on barley leaves. These data are consistent with proteasome mediated protein turnover being required for infection related development and pathogenicity in $M$. oryzae.

\section{Components of Ubiquitin Mediated Protein Modification} are Highly Conserved and Regulated in Response to Developmental and Nutritional Stimuli in M. oryzae

To assess the degree of conservation of proteins associated with ubiquitin mediated protein modification, we conducted an InterPro domain search. One hundred and eighty three proteins putatively involved in ubiquitination pathway in $M$. oryzae were identified (Table 1, Table S2). Forty four proteins directly related to ubiquitin (UA) were found including 12 containing ubiquitin and 13 with an ubiquitin interacting motif. Eight proteins were ubiquitin-activating enzymes, E1, and 21 proteins were ubiquitin- 
A

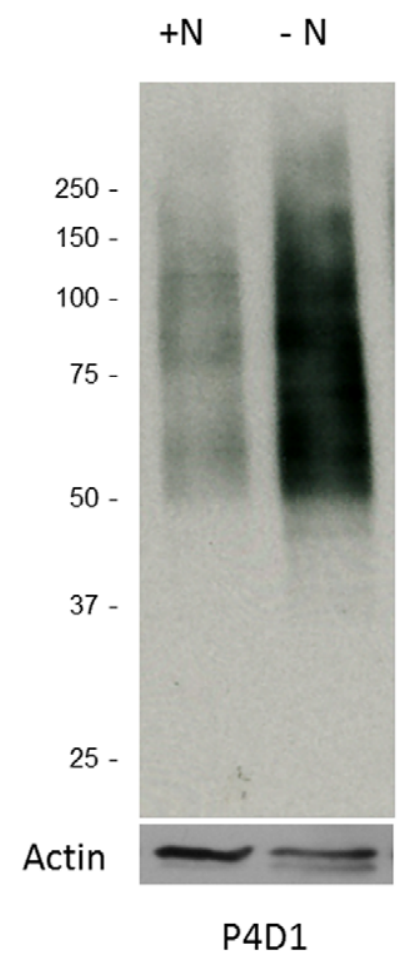

B

\section{WT $\quad$ MT1 EC $\quad$ MT2}

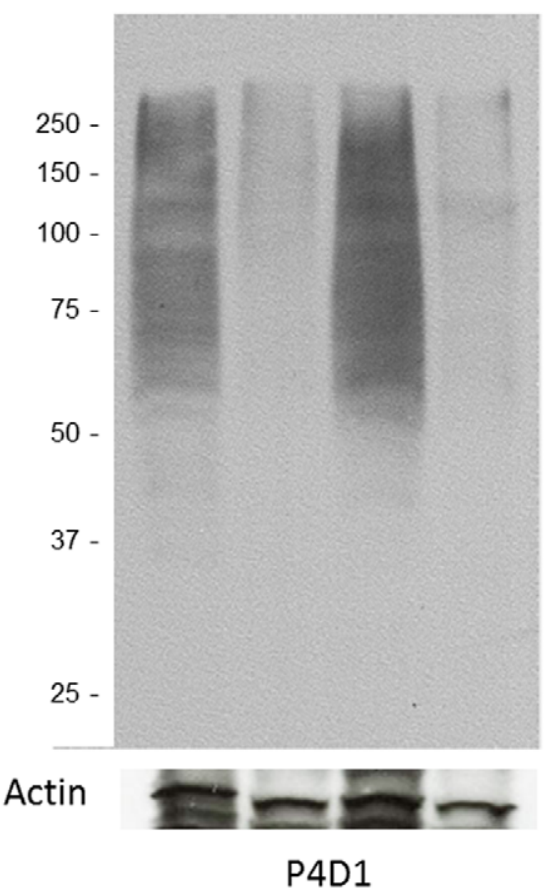

WT MT1 EC MT2

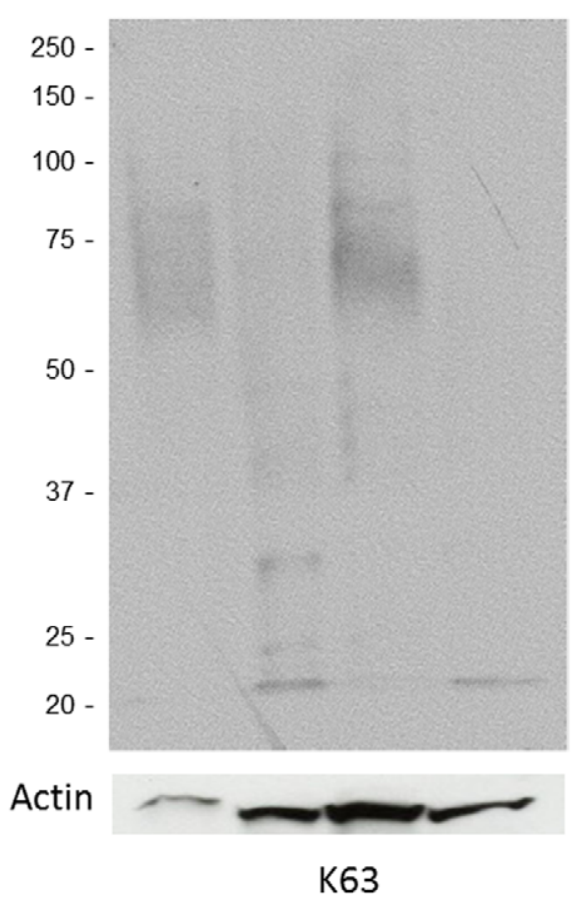

Figure 4. Protein ubiquitination is induced by nitrogen starvation and requires the polyubiquitin gene, MGG_01282. A. 70$15 M$. oryzae was grown in liquid minimal medium with $(+\mathrm{N})$ and without $(-\mathrm{N})$ nitrogen sources. Protein extracts from each sample were probed with an antibody recognizing both ubiquitin and polyubiquitin (P4D1). An anti-actin antibody was used to compare the relative amount of total proteins in each lane. B and C. Wild type (WT), MGG_1282 deletion mutants (MT1, MT2) and ectopic (EC) strains were incubated in minimal medium. Protein extracts from each sample were probed with antibodies recognizing ubiquitin and polyubiquitin (P4D1) or specifically polyubiquitin (K63), respectively. Panel $B$ were exposed to $X$ ray film for a longer compared to panel $A$.

doi:10.1371/journal.pone.0042868.g004

conjugating enzymes, E2. The most diverse and largest group was ubiquitin ligase, E3, which had 94 members and included 53 zinc finger, ring-type (IPR001841) domain containing proteins. Components of multi-protein E3 ubiquitin ligase Skp, Cullin and F-box containing complex (SCF complex) were found and included one Skp, four Cullins and 26 F-box containing proteins. A total of 20 proteins were identified as proteins for deubiquitination.

In previous work, we conducted extensive microarray analysis $[13,19]$. Further inspection of the data revealed that 161 of the 183 ubiquitin pathway genes were expressed during conidia germination, appressorium formation or nitrogen starvation (Figure 2, Table S2). The most dramatic changes in gene expression occurred during conidia germination, where $87.6 \%$ of the genes (141 out of 161) showed at least a $50 \%$ increase in transcript levels and $39 \%$ were significantly induced $(>2$ fold up, $\mathrm{p}<0.05)$. This is in contrast to the entire transcriptome, where only $21 \%(2,087$ of $10,176)$ of genes were significantly $(>2$ fold up, $\mathrm{p}<0.05)$ induced. Sixty three percent (5 out of 8 ) of genes encoding ubiquitinactivating enzyme, E1, 42\% (8 out of 19) of ubiquitin-conjugating enzyme, E2, 29\% of (23 out of 79) ubiquitin ligase, E3 and 39\% (7 out of 18) of de-ubiquitinating enzyme showed increased gene expression. No ubiquitin pathway genes exhibited significant down regulation during conidia germination, except for MGG_01282. Transcripts of MGG_01282, predicted to encode a polyubiquitin protein, were significantly more abundant in intact conidia compared with germinating cells.
Contrary to the conidia germination, transcript levels of the majority of ubiquitin associated proteins did not change significantly during appressorium formation. Only 3.7\% (6) and 5.0\% (8) of genes were induced during appressorium induction in response to hydrophobic surface signal and cyclic AMP, respectively, and $0.6 \%(1)$ and $2.5 \%$ (4) genes were down regulated. Transcripts of MGG_01282, a polyubiquitin protein and MGG_07127, an autophagy-related E1 like protein were more abundant during appressorium formation compared to germinating conidia. No ubiquitin-conjugating enzyme, E2, genes were differentially expressed. Among 79 ubiquitin ligases, E3, 4\% (3) and 8\% (6) genes were induced by physical and chemical signals and only one gene, MGG_10932 was down regulated. Two F-box proteins, MGG_07785 and MGG_08019, were up regulated by both signals. MGG_08638, a ubiquitin C-terminal hydrolase was the sole de-ubiquitination gene that was induced by the hydrophobic physical cue.

Examination of previous data under nitrogen starvation conditions revealed transcriptional induction of about 9\% (14) and $11 \%(18)$ of ubiquitin pathway genes, and $3 \%(4)$ and $2 \%$ (3) with reduced expression of $12 \mathrm{hr}$ and $48 \mathrm{hr}$, respectively. Two F-box containing proteins (MGG_00768 and MGG_04395) and one zinc finger, ring-type E3 ligase (MGG_02837) were induced at both $12 \mathrm{hr}$ and $48 \mathrm{hr}$. The expression of only 2 genes was up-regulated by appressorium inducing physical and chemical signals as well as by nitrogen starvation. Transcript levels of MGG_01282 were significantly 
A
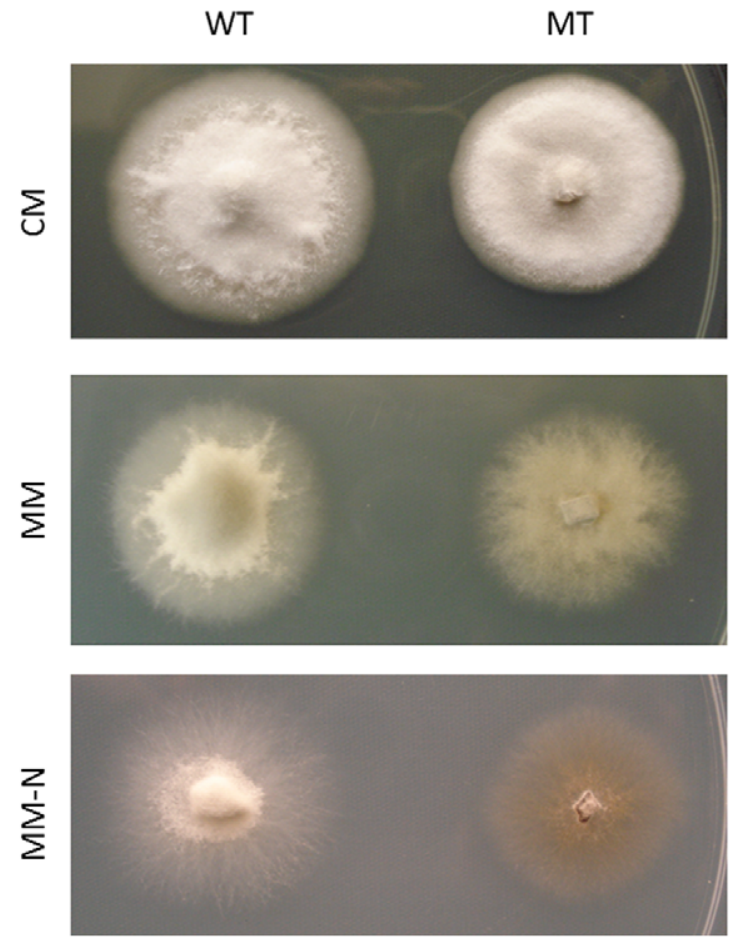

B

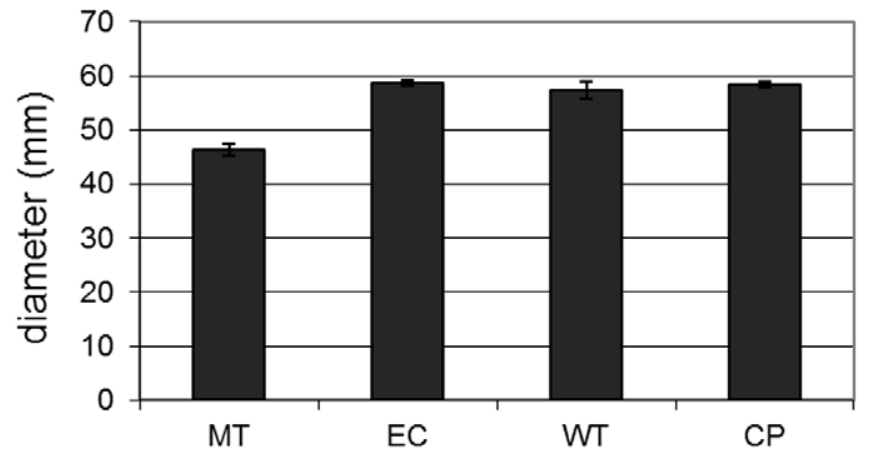

Figure 5. MGG_01282 is required for normal vegetative growth. A. 70-15 wild type (WT) and mutant strain (MT) were incubated for 7 days on complete medium (CM), minimal medium (MM) and minimal medium without nitrogen source (MM-N). B. Radial mycelial growths were measured in mutant stain MT1 and MT2 and were compared to those of ectopic (EC), WT and MGG_012982 complemented strain (CP). doi:10.1371/journal.pone.0042868.g005

elevated during appressorium maturation (3.7 fold) and cyclic AMP induction (3.0 fold) as well as with nitrogen starvation for $12 \mathrm{hr}$ (8.2 fold) and $48 \mathrm{hr}$ (2.4 fold). Expression of the F-box protein, MGG_07785 was induced during appressorium formation and at $12 \mathrm{hr}$ nitrogen starvation. In sum, transcription levels of a large proportion (39\%) of genes associated with the ubiquitination pathway were induced during conidia germination. However, only a small proportion (between 5-11\%) was induced during appressorium formation or in response to nitrogen starvation. Very few genes were induced under both conditions. One of the notable exceptions was the gene encoding polyubiquitin, MGG_01282.

\section{Highly Conserved but Structurally Diverse Ubiquitin Genes in M. oryzae Show Different Expression Patterns during Development and Starvation}

In addition to MGG_01282, which encodes a protein with 4 ubiquitin monomers, we identified 11 other proteins in $M$. oryzae that contain single or partial ubiquitin motifs. Some of which contained other functional domains that are related to the ubiquitination pathway (Figure 3A). MGG_09887 encodes a single ubiquitin monomer, whereas other single ubiquitin proteins MGG_06044 and MGG_07928 contain large and small ribosomal subunits at their $\mathrm{C}$ terminals, respectively. Among the proteins that contain a partial ubiquitin moiety, MGG_01318 and MGG_01656 possess an ubiquitin associated domain and MGG_02970 has a ubiquitin carboxyl-terminal hydrolase domain at the $\mathrm{C}$ terminal region. Two proteins, MGG_05137 and MGG_05737 contain an ubiquitin-like SUMO domain at their $\mathrm{C}$ terminal and $\mathrm{N}$ terminal regions, respectively.
Except for MGG_01282, expression of all ubiquitin containing genes were induced during conidia germination, however, no significant change was observed during appressorium induction and nitrogen starvation in most cases (Figure 3). The exceptions were MGG_07928 and MGG_06044, which were down-regulated during both conditions. Gene expression of MGG_09887, encoding a single ubiquitin moiety, was induced under nitrogen starvation. Because MGG_01282 showed the most dynamic changes in gene expression, being highly induced during appressorium formation and nitrogen starvation, it was subjected to further examination.

\section{Nitrogen Starvation Results in a Dramatic Increase in Protein Ubiquitination which is Mediated by the Polyubiquitin Protein, MGG_01282}

Protein ubiquitination is known to be directly linked to the cellular nutrient status [25]. To examine this relationship in $M$. oryzae, we investigated the correlation between ubiquitination and one of the major developmental signals, nitrogen starvation. As shown by immunoblots with antibodies recognizing polyubiquitin and ubiquitin in Figure $4 \mathrm{~A}$, when the fungus was exposed to the nitrogen limiting conditions for $12 \mathrm{hr}$, protein ubiquitination dramatically increased compared to the condition without nitrogen stress. To further investigate the function of MGG_01282, we generated deletion mutants using standard protocols [19]. Immunoblot analyses of proteins extracted from growth on minimal medium or under nitrogen starvation showed mutant strains contained strikingly less ubiquitinated proteins compared to wild type and ectopic strains (Figure 4B). In addition, immunoblot analyses using the linkage specific 
A

WT

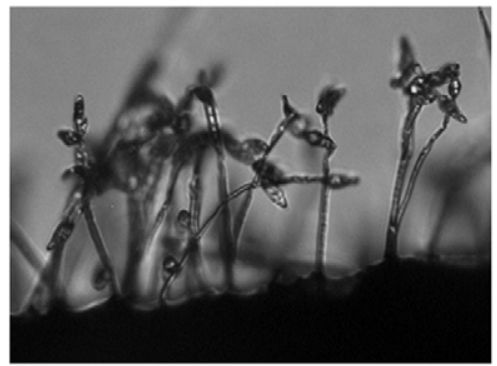

MT

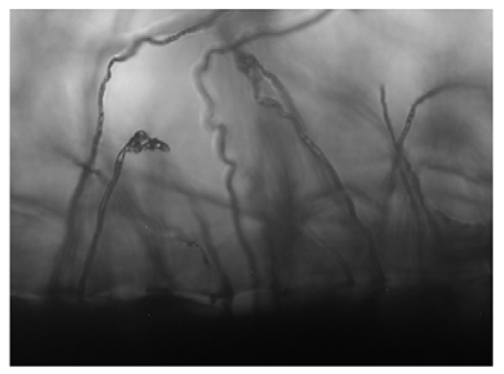

EC

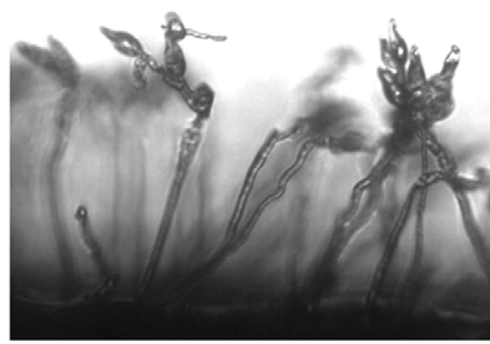

B

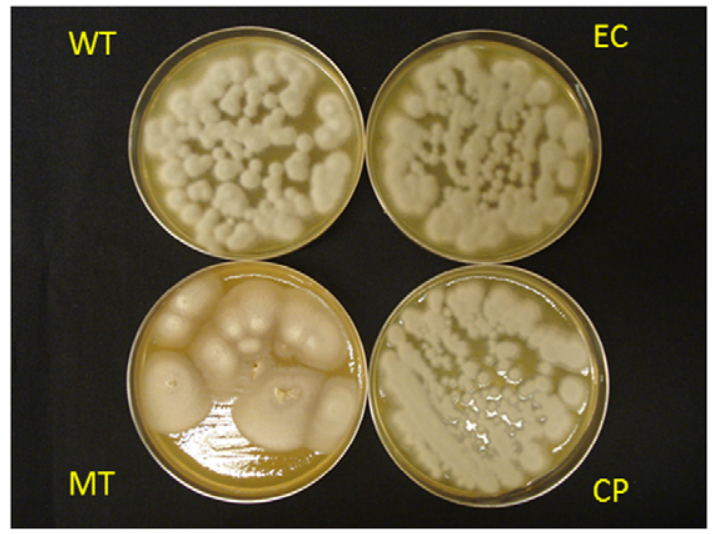

C

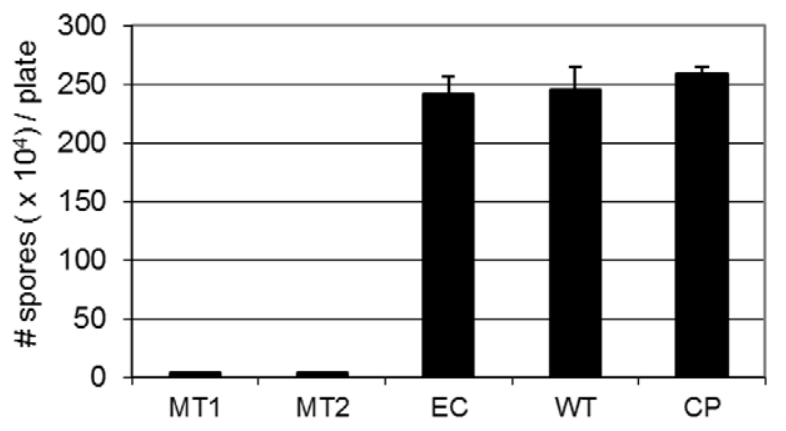

Figure 6. MGG_01282 is required for normal conidia production. 70-15 wild type (WT), MGG_01282 deletion mutants (MT1, MT2), ectopic (EC) and MGG_01282 complemented (CP) strains were incubated on V8 medium (A, B) and the average number of conidia produced 10 days after inoculation (C).

doi:10.1371/journal.pone.0042868.g006

antibody (K63) revealed mgg_01282 deletion mutants contained dramatically reduced K63 linked polyubiquitin targets. K63 ubiquitin targets are known to be involved in cellular development and signal transduction in eukaryotic cells [26]. In summary, protein ubiquitination was significantly elevated under nitrogen starvation and was primarily mediated by polyubiquitin protein MGG_01282.

\section{The Polyubiquitin Protein MGG_01282 is Essential for Fungal Growth, Development and Pathogenicity}

To investigate the biological role of polyubiquitin in $M$. oryzae, we compared phenotypes of the knockout mutant with ectopic and wild type strains as well as with a strain complemented with MGG_01282. Deletion of MGG_01282 resulted in significant changes in fungal growth, morphology and development in $M$. oryzae. Radial growth of mutants on solid nutrient rich complete medium or minimal medium was significantly retarded (about 17\%) and aerial hyphae was suppressed compared with the wild type. This growth reduction was more severe under nitrogen limiting conditions (Figure 5A and $5 \mathrm{~B})$.

MGG_01282 mutants also showed defects in conidiation. The number of conidia generated by the mutants after 7 day incubation on V8 medium was significantly reduced (more than $90 \%$ ) compared to the wild type (Figure 6). In addition, while most conidia ( $\geq 94 \%$ ) from wild type consisted of three cells, divided by two septa, about half of mutant conidia contained single $(35 \%)$ or no septa $(13 \%)$ (Figure 7A). Mutant conidia were smaller and more rounded than those produced by the wild type strain (Figure 7B). Ectopic and complemented strains were indistinguishable from the wild type for growth, conidiation and conidia morphology (Figures 5, 6, and 7).

Almost all wild type and ectopic conidia germinated on hydrophobic surface and about $90 \%$ of them successfully developed appressoria after $24 \mathrm{hr}$ incubation. In mutant strains, $62 \%$ of conidia germinated but most failed to develop appressoria (Figure $8 \mathrm{~A}$ and $8 \mathrm{~B}$ ). In addition, mutant appressoria were less pigmented and unable to cause disease. When sprayed on barley and rice leaves, mutant strains produced no disease symptoms (Figure 8C). To investigate whether MGG_01282 mediated growth in planta, barley leaves were inoculated onto pinprick wounded sites. MGG_01282 deletion mutants showed no blast symptoms on wounded plants (Figure 8D). Taken together, these data indicate that the polyubiquitin gene, MGG_01282 is essential for conidiation, appressorium formation and invasive growth in planta.

M. oryzae 70-15 is heterothallic and requires an opposite mating type strain for sexual reproduction. In this study, we crossed the wild type 70-15, MGG_01282 deletion mutant, ectopic and complemented strains with strain 4091-5-8. Four weeks after crossing, wild type, ectopic and complemented strains developed melanized perithecia, which contained ascoconidia within the asci (Figure 9). In these crosses, all strains were female fertile and two rows of perithecia were clearly visible (Figure 9). In crosses with MGG_01282 deletion mutant, the number of perithecia was reduced. Within 4091-5-8 tissue, 
A
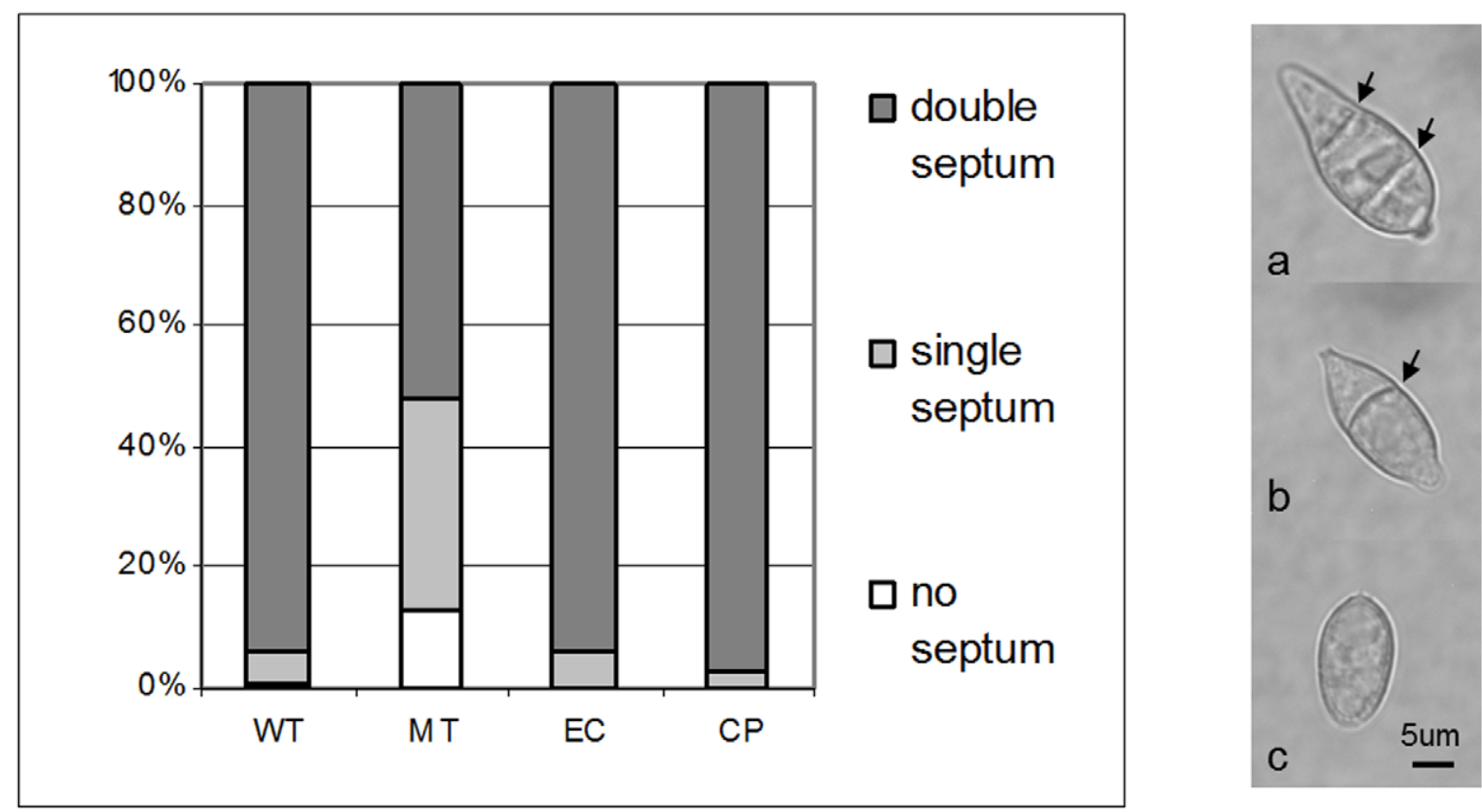

B

\begin{tabular}{|c|c|c|c|}
\hline Strain & \# septum & conidia length (um) & conidia ore width (um) \\
\hline WT & $1.9 \pm 0.3$ & $24.9 \pm 2.6$ & $10.3 \pm 0.9$ \\
\hline MT & $1.4 \pm 0.7$ & $23.8 \pm 5.6$ & $8.6 \pm 1.3$ \\
\hline EC & $1.9 \pm 0.2$ & $24.9 \pm 2.5$ & $10.0 \pm 1.1$ \\
\hline CP & $2.0 \pm 0.2$ & $25.0 \pm 1.6$ & $9.6 \pm 0.9$ \\
\hline
\end{tabular}

Figure 7. MGG_01282 deletion mutants produced abnormal conidia. A. For each strain, conidia collected from V8 medium were grouped according to the number of septa, double (a); single (b) or no septa (arrow marked). The percentage of each group per strain is presented. B. Average number of septa, conidia length and width is presented.

doi:10.1371/journal.pone.0042868.g007

typical asci were observed. However, within the deletion mutant tissue only highly condensed and melanized fungal structures were detected that failed to further develop into perithecia (Figure 9). These data indicate that MGG_01282 is required for normal sexual development.

\section{Ubiquitinated Protein Profiling during Fungal Growth Under Nitrogen Stress}

We found that protein ubiquitination is highly increased during nitrogen starvation. This suggests that when nitrogen is limiting, $M$. oryzae actively recycles cellular materials and redirects biological pathways to cope with the nitrogen deficiency for survival, at least part of which is mediated by protein ubiquitination. To identify proteins targeted for ubiquitination under nitrogen stress, we incubated wild type $M$. oryzae in minimal media without a nitrogen source for $18 \mathrm{hr}$ and enriched polyubiquitinated proteins using agarose TUBE2. TUBE2 contains a protein with very high affinity for polyubiquitinated proteins [27]. As a negative control, we used agarose lacking the affinity protein. We found the enrichment to be effective as evidenced by protein staining and immunoblot analysis of the enriched protein fraction (Figure S2). Mass spectral analysis revealed 63 proteins to be unique or significantly enriched in the affinity purified sample compared to negative control (Table S3). As expected, ubiquitin was found to be the most abundant protein based on spectral counts. Based on gene ontology using BLAST2GO [28], compared to the entire proteome, proteins involved in translation $(30.2 \%)$, metabolic process $(17.4 \%)$, transport $(14.3 \%)$, and protein metabolic process $(9.5 \%)$ including ubiquitin proteins 
A
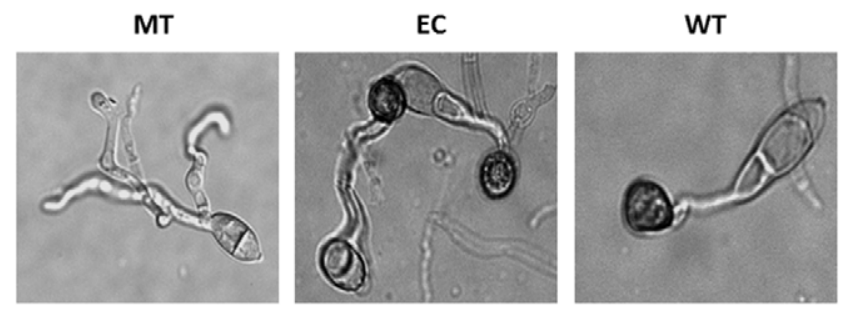

B

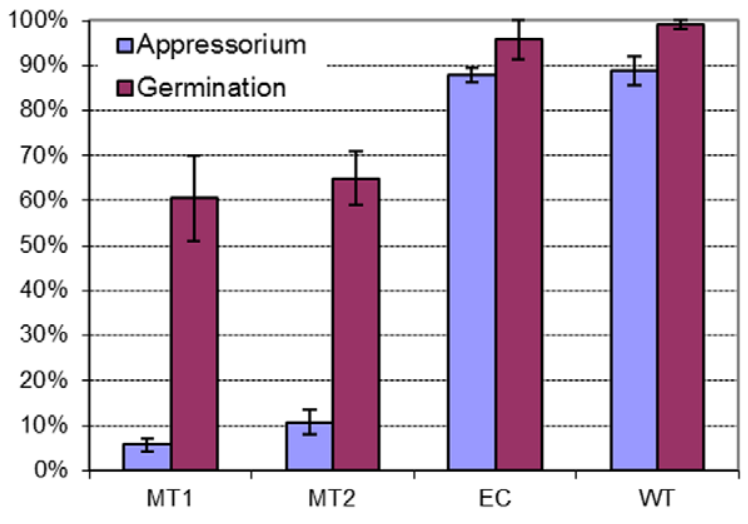

C

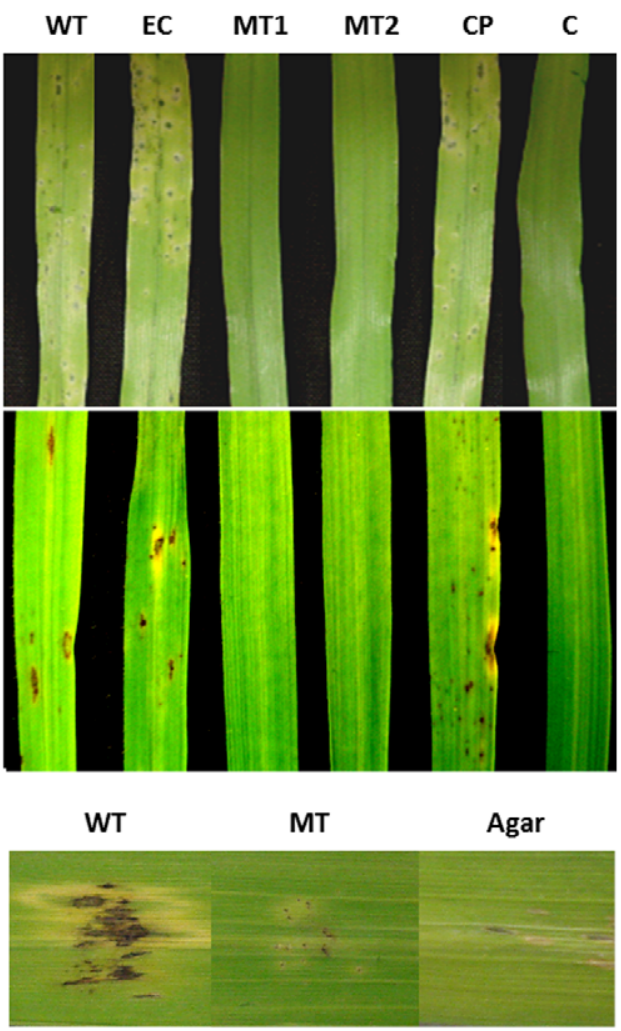

Figure 8. MGG_01282 is required for appressorium formation and pathogenicity in rice and barley. A. Germination and appressorium formation of conidia from 70-15 wild type (WT), MGG_01282 deletion mutants (MT1, MT2) and ectopic (EC) strains on a hydrophobic surface after $24 \mathrm{hr}$ incubation. B. Percentage of conidia germination and appressorium formation from at least 100 conidia per replicate with 3 replicates per strain. C. Disease development of each strain including a complemented (CP) strain inoculated onto barley (upper panel) and rice (lower panel) seedlings. Disease progress was evaluated compared to water treated control (C) 5 days after inoculation. D. Wound assay on detached barley leaves. Barley leaves were wounded by making a tiny pinprick with a sterile needle. Leaves were inoculated with 8 day old V8 agar block of each strain and incubated in a humid chamber. Disease progress was evaluated compared to control agar and photographed 5 days post-inoculation. doi:10.1371/journal.pone.0042868.g008

and components of the proteasome were over-represented (Table S3). Several proteins associated with cytoskeleton and stress responses including actin, tubulins and heat shock proteins were also identified (Table S3). We further identified proteins predicted to be involved repression of carbon catabolism such as homologs of a hulA E3 ligase (MGG_07255) and as arrestin containing protein (MGG_01045) as well as proteins involved in cell signaling including MGG_01588 and MGG_13806, both 14-3-3 proteins.

\section{Discussion}

The availability of the entire genome sequence and global gene expression profiles for $M$. oryzae has enabled new insight into infection related development and pathogenicity [19,29]. Our previous studies revealed a hitherto unknown link between protein degradation and infection structure development in M. oryzae [19]. Genes required for the non-selective protein degradation process referred to as autophagy have been characterized and shown to be important for fungal pathogenicity in M. oryzae [30,31]. On the other hand, prior to this study, little direct evidence was available linking the highly selective protein degradation process, mediated by ubiquitin, to fungal pathogenicity.

To establish a link between protein turnover through the ubiquitin-proteasome complex and fungal pathogenicity, we first demonstrated that treatment with the $26 \mathrm{~S}$ proteasome inhibitor,
Bortezomib, resulted in a significant delay in germination and appressorium formation. Moreover, addition of Bortezomib to inoculum blocked symptom development, even though melanized appressoria were formed after $48 \mathrm{hr}$. A recent report, which was published during the course of this research, also showed the proteasome inhibitors MG-132, proteasome inhibitor I and proteome inhibitor II delayed conidia germination and appressorium formation in $M$. oryzae as well as suppressed infection of rice leaves [32].

In order to elucidate the underlying mechanisms, we then explored the machinery associated with ubiquitin mediated protein turnover. Similar to other eukaryotic organisms [20], many of the components are highly conserved in $M$. oryzae. Examination of whole genome microarray gene expression data further revealed that most of the components for protein ubiquitination were induced upon conidia germination but did not dramatically change expression during appressorium formation. The elevated expression of genes associated with ubiquitination during germination in the absence of external nutrients likely reflects the cells preparation for recycling proteins and other storage components. A few genes were observed to be induced during appressorium induction and nitrogen starvation but none of them had been previously characterized. Interestingly, we discovered that a polyubiquitin encoding gene MGG_01282 was most highly expressed in intact conidia rather than in germinating 


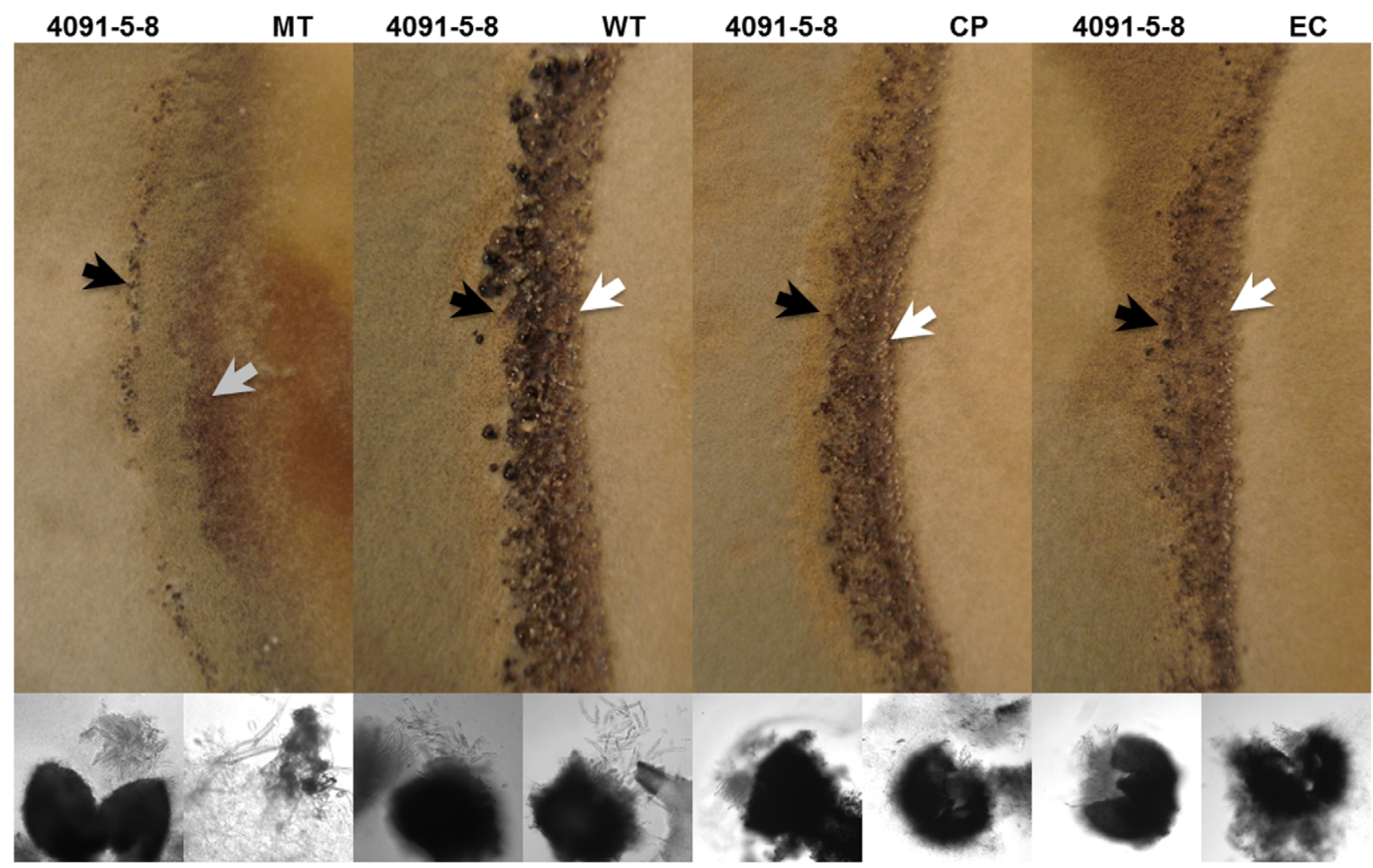

Figure 9. MGG_01282 deletion mutants are female sterile. 70-15 wild type (WT), MGG_01282 deletion mutant (MT), ectopic (EC) and MGG_01282 complemented (CP) strains were crossed with the opposite mating type, 4091-5-8 strain, on oat meal media. Fruiting bodies formed in each test strain and 4091-5-8 strain are marked with white and black arrows respectively. Below are shown corresponding perithecia and erupting ascospores. The melanized structure in MT is indicated by a gray arrow with corresponding enlargement below.

doi:10.1371/journal.pone.0042868.g009

conidia and was significantly induced during appressorium formation and nitrogen starvation.

In fungi, polyubiquitin does not appear to be an essential gene, although it likely provides the main supply of cellular ubiquitin protein in response to developmental and environmental stimuli. Here, we showed by Western blot analysis that protein ubiquitination is highly induced under nitrogen starvation and through examination of the knock-out mutant that protein ubiquitination is mainly mediated by MGG_01282 gene products. The gene deletion mutant in $M$. oryzae, although viable, exhibited numerous phenotypic detects, including defects in mycelia growth, conidia morphology, sexual reproduction, infection structure development and pathogenicity. Similar types of defects have been observed in Saccharomyces cerevisiae and C. albicans. Loss of UBI4 in S. cerevisiae resulted in increased sensitivity to starvation and to amino acid analogs as well as reduced growth at high temperatures [33-35]. UBI4 deletion mutant of C. albicans grew relatively normally on rich media, but displayed morphological and cell cycle defects when exposed to a number of stresses including temperature, peroxide and several anti-fungal drugs that interfere with cell wall biosynthesis [9]. In $M$. oryzae, the polyubiquitin deletion mutant although growing relatively poorly on all media, was most affected under nitrogen starvation. These data are consistent with ubiquitination playing a major role in protein turnover required for normal growth and development under a variety of stress conditions.

Examination of the 63 proteins unique or significantly enriched following affinity purification for polyubiquitination revealed that the most abundant group of proteins was components of the ribosome. Other studies have also shown that proteins associated with ribosome are ubiquitinated [9,36]. As may be expected under nitrogen starvation, translation is curtailed and the machinery is recycled via ubiquitination and proteasome mediated degradation. We also identified other proteins including heat shock proteins and a succinate dehydrogenase, which in C. albicans showed increased ubiquitination in response to heat and oxidative stress [9]. In a global analysis of ubiquitination in the human cell line HEK293, 236 proteins were identified to be ubiquitinated [37]. Among these, we found 20 that matched $M$. oryzae proteins in our ubiquitinated protein data set and these included heat shock proteins, actin and tubulins and ribosomal proteins. These data suggest a certain level of conservation of proteins targeted for ubiquitination across kingdoms.

It was noteworthy that two of the proteins targeted by ubiquitin identified in our data set were a HECT domain containing E3 ligase, MGG_07255 and an arrestin domain containing protein MGG_01045, orthologs of Rsp5 and Rod1 in $S$. cerevisiae respectively. Rsp5 is known to be involved in a variety of cellular process including endocytosis, multivesicular body sorting and RNA stability [38-40]. The arrestin-like adaptor, Rodl binds to Rsp5 and mediates ubiquitination and endocytic internalization of membrane transporters, which are degraded in the vacuole. In $A$ nidulans, CreD, an ortholog of Rodl, has been shown to control carbon catabolite repression with possible interaction with HECT ligase, HulA [41]. Selfubiquitination of other HECT ligase ortholog NEDD4-1, 
NEDD4-2 is regulated through intramolecular interaction between the WW domains and PY motifs in HECT domains $[42,43]$. This suggests that ubiquitination and following degradation of MGG_07255 is tightly regulated depending upon environmental conditions. During nitrogen starvation, ubiquitination of MGG_07255/MGG_01045 could be important for reprogramming fungal cells to cope with nutrient limiting conditions.

Over the past twenty years, the role of protein ubiquitination in eukaryotic cells has been emerging, however, relatively little is known related to fungal development, pathogenicity and disease control. Here, we showed through a combination of pharmacological, molecular and proteomic analysis that ubiquitin mediated posttranslational modification is a central regulator in fungal nutrition, development and pathogenicity. Future study will be focused on specific components of the ubiquitination processes including identification of specific ubiquitination target proteins, which may offer up novel strategies for plant disease control.

\section{Supporting Information}

Figure S1 Targeted knock out of the polyubiquitin gene (MGG_01282) in M. oryzae. A. A gene replacement cassette in which the hygromycin resistance gene $(\mathrm{HPH})$ was flanked with about $1 \mathrm{~kb}$ each upstream and downstream of the MGG_01282 gene was constructed and transformed into wild type cells. Through homologous recombination, the MGG_01282 gene was replaced with HPH generating MGG_01282 deletion mutants. B. Deletion mutants were confirmed by Southern blot analysis using right flanking sequences $(*)$ as a probe. Absence of Xho I restriction enzyme recognition site $(\mathrm{X})$ in $\mathrm{HPH}$ resulted in a larger labeled fragment in the mutant which was distinct from that of wild type.

(TIF)

\section{References}

1. Hershko A, Ciechanover A (1998) The ubiquitin system. Annu Rev Biochem 67: 425-479.

2. Takada K, Hibi N, Tsukada Y, Shibasaki T, Ohkawa K (1996) Ability of ubiquitin radioimmunoassay to discriminate between monoubiquitin and multiubiquitin chains. Biochim Biophys Acta 1290: 282-288.

3. Ryu KY, Baker RT, Kopito RR (2006) Ubiquitin-specific protease 2 as a tool for quantification of total ubiquitin levels in biological specimens. Anal Biochem 353: $153-155$.

4. Ozkaynak E, Finley D, Varshavsky A (1984) The yeast ubiquitin gene - head to-tail repeats encoding a polyubiquitin precursor protein. Nature 312: 663-666.

5. Ozkaynak E, Finley D, Solomon MJ, Varshavsky A (1987) The yeast ubiquitin genes - a family of natural gene fusions. EMBO J 6: 1429-1439.

6. Pieterse CMJ, Risseeuw EP, Davidse LC (1991) An in planta induced gene of Phytophthora Infestans codes for ubiquitin. Plant Mol Biol 17: 799-811.

7. McCafferty HRK, Talbot NJ (1998) Identification of three ubiquitin genes of the rice blast fungus Magnaporthe grisea, one of which is highly expressed during initial stages of plant colonisation. Curr Genet 33: 352-361.

8. Loser K, Weltring KM (1998) Induction of a polyubiquitin gene (ubi1) by potato phytoalexins and heat shock in Gibberella pulicaris. Curr Genet 34: 404-409.

9. Leach MD, Stead DA, Argo E, MacCallum DM, Brown AJP (2011) Molecular and proteomic analyses highlight the importance of ubiquitination for the stress resistance, metabolic adaptation, morphogenetic regulation and virulence of Candida albicans. Mol Microbiol 79: 1574-1593.

10. Ou SH (1980) Pathogen variability and host-resistance in rice blast disease. Annu Rev Phytopathol 18: 167-187.

11. Howard RJ, Valent B (1996) Breaking and entering: Host penetration by the fungal rice blast pathogen Magnaporthe grisea. Annu Rev Microbiol 50: 491-512.

12. Lee YH, Dean RA (1993) cAMP regulates infection structure formation in the plant-pathogenic fungus Magnaporthe grisea. Plant Cell 5: 693-700.

13. Donofrio NM, Oh Y, Lundy R, Pan H, Brown DE, et al. (2006) Global gene expression during nitrogen starvation in the rice blast fungus, Magnaporthe grisea. Fungal Genet Biol 43: 605-617.

14. Xu JR, Hamer JE (1996) MAP kinase and cAMP signaling regulate infection structure formation and pathogenic growth in the rice blast fungus Magnaporthe grisea. Genes Dev 10: 2696-2706.
Figure S2 Enrichment of ubiquitinated proteins using TUBE2 (Tandem Ubiquitin Binding Entity 2). $85 \mathrm{ug}$ of $M$. oryzae total proteins were extracted from liquid minimal media without nitrogen sources and was subjected to pulldown with Agarose-TUBE2. Input (I), unbound(U), eluted (E) and wash (W) samples were load to 4-20\% gradient gel (Invitrogen) along with protein marker(M). Following electrophoresis, gel was stained with coomassie blue. After blotting, membrane was stained with ponceau and was probed with anti-ubiquitin antibody. Compared to the amount of total proteins, ubiquitinated proteins were found to be highly enriched in the eluted sample.

(TIF)

Table S1 Primer sequences for gene specific replacement cassette construction.

(XLS)

Table S2 List of ubiquitin pathway associated proteins in M.oryzae and gene expression profiling during conidia germination, appressorium formation and under nitrogen starvation. (XLSX)

Table S3 List of putative ubiquitinated proteins during nitrogen starvation in $M$. oryzae.

(XLSX)

\section{Acknowledgments}

We thank Dr. Sudha K. Shenoy at Duke University for conducting Western blot analysis.

\section{Author Contributions}

Conceived and designed the experiments: YO RAD. Performed the experiments: YO WF SH AS EG. Analyzed the data: YO RAD. Contributed reagents/materials/analysis tools: DM. Wrote the paper: YO RAD.

15. Nguyen QB, Kadotani N, Kasahara S, Tosa Y, Mayama S, et al. (2008) Systematic functional analysis of calcium-signalling proteins in the genome of the rice-blast fungus, Magnaporthe oryzae, using a high-throughput RNA-silencing system. Mol Microbiol 68: 1348-1365.

16. deJong JC, McCormack BJ, Smirnoff N, Talbot NJ (1997) Glycerol generates turgor in rice blast. Nature 389: 244-245.

17. Veneault-Fourrey C, Barooah M, Egan M, Wakley G, Talbot NJ (2006) Autophagic fungal cell death is necessary for infection by the rice blast fungus. Science 312: 580-583.

18. Saunders DGO, Aves SJ, Talbot NJ (2010) Cell cycle mediated regulation of plant infection by the rice blast fungus. Plant Cell 22: 497-507.

19. Oh Y, Donofrio N, Pan HQ Coughlan S, Brown DE, et al. (2008) Transcriptome analysis reveals new insight into appressorium formation and function in the rice blast fungus Magnaporthe oryzae. Genome Biol 9: R85.

20. Semple CAM (2003) The comparative proteomics of ubiquitination in mouse. Genome Res 13: 1389-1394.

21. Reid RJD, Lisby M, Rothstein R (2002) Cloning-free genome alterations in Saccharomyces cerevisiae using adaptamer-mediated PCR. Methods Enzymol 350: 258-277.

22. Sweigard JA, Chumley FG, Valent B (1992) Disruption of a Magnaporthe grisea cutinase gene. Mol Genet Genomics 232: 183-190.

23. Valent B, Farrall L, Chumley FG (1991) Magnaporthe grisea genes for pathogenicity and virulence identified through a series of backcrosses. Genetics 127: 87-101.

24. Gokce E, Shuford C, Franck W, Dean R, Muddiman D (2010) Evaluation of normalization methods on GeLC-MS/MS label-free spectral counting data to correct for variation during proteomic workflows. J Am Soc Mass Spectrom 22: 2199

25. Finn PF, Dice JF (2006) Proteolytic and lipolytic responses to starvation. Nutrition 22: 830 .

26. Passmore LA, Barford D (2004) Getting into position: the catalytic mechanisms of protein ubiquitylation. Biochem J 379: 513-525.

27. Hjerpe R, Aillet F, Lopitz-Otsoa F, Lang V, England P, et al. (2009) Efficient protection and isolation of ubiquitylated proteins using tandem ubiquitinbinding entities. EMBO Rep 10: 1250. 
28. Gotz S, Garcia-Gomez JM, Terol J, Williams TD, Nagaraj SH, et al. (2008) High-throughput functional annotation and data mining with the Blast2GO suite. Nucleic Acids Res 36: 3420-3435.

29. Dean RA, Talbot NJ, Ebbole DJ, Farman ML, Mitchell TK, et al. (2005) The genome sequence of the rice blast fungus Magnaporthe grisea. Nature 434: 980986.

30. Liu XH, Liu TB, Lin FC (2008) Monitoring autophagy In Magnaporthe oryzae. Methods in Enzymology 451: 271-294.

31. Kershaw MJ, Talbot NJ (2009) Genome-wide functional analysis reveals that infection-associated fungal autophagy is necessary for rice blast disease. Proc Natl Acad Sci U S A 106: 15967-15972.

32. Wang Y, Kim SG, Wu J, Yu S, Kang KY, et al. (2011) Proteasome inhibitors affect appressorium formation and pathogenicity of the rice blast fungus, Magnaporthe oryzae. Plant Pathol J 27: 225-231.

33. Tanaka K, Matsumoto K, Tohe A (1988) Dual regulation of the expression of the polyubiquitin gene by cyclic-amp and heat-shock in yeast. Embo J 7: 495502.

34. Fraser J, Luu HA, Neculcea J, Thomas DY, Storms RK (1991) Ubiquitin gene expression: response to environmental changes. Curr Genet 20: 17

35. Finley D, Ã-zkaynak E, Varshavsky A (1987) The yeast polyubiquitin gene is essential for resistance to high temperatures, starvation, and other stresses. Cell 48: 1035-1046.
36. PengJ, Schwartz D, Elias JE, Thoreen CG, Cheng D, et al. (2003) A proteomics approach to understanding protein ubiquitination. Nature Biotechnol 21: 921926.

37. Xu G, Paige JS, Jaffrey SR (2010) Global analysis of lysine ubiquitination by ubiquitin remnant immunoaffinity profiling. Nature Biotechnol 28: 868.

38. Shcherbik N, Pestov DG (2011) The ubiquitin ligase Rsp5 is required for ribosome stability in Saccharomyces cerevisiae. RNA 17: 1422-1428.

39. Katzmann DJ, Sarkar S, Chu T, Audhya A, Emr SD (2004) Multivesicular body sorting: Ubiquitin ligase Rsp5 is required for the modification and sorting of carboxypeptidase S. Mol Biol Cell 15: 468-480.

40. Belgareh-Touze N, Leon S, Erpapazoglou Z, Stawiecka-Mirota M, UrbanGrimal D, et al. (2008) Versatile role of the yeast ubiquitin ligase Rsp5p in intracellular trafficking. Biochem Soc Trans 36: 791-796.

41. Boase NA, Kelly JM (2004) A role for creD, a carbon catabolite repression gene from Aspergillus nidulans, in ubiquitination. Mol Microbiol 53: 929-940.

42. Gallagher E, Gao M, Liu Y-C, Karin M (2006) Activation of the E3 ubiquitin ligase Itch through a phosphorylation-induced conformational change. Proc Natl Acad Sci U S A. 1717-1722.

43. Bruce MC, Kanelis V, Fouladkou F, Debonneville A, Staub O, et al. (2008) Regulation of Nedd4-2 self-ubiquitination and stability by a PY motif located within its HECT-domain. Biochem J 415: 155-163. 